\title{
PARAMETRIC STUDY IN SURFACE FINISHING OF INCONEL 718 SURFACE WITH MAGNETIC ABRASIVE FINISHING PROCESS
}

\section{LOVEPREET SINGH, HARISH KUMAR \& ARUN KUMAR}

Department of Mechanical Engineering, Chandigarh University, Gharuan, Mohali, Punjab India

Inconel 718 is a nickel based alloy, used in high pressure and temperature applications, due to its high strength and thermal resistance properties. Machining of Inconel 718 is very difficult due to its toughness. Surface finishing of Inconel 718 is also a difficult task to deal with. In the present work, the magnetic, abrasive finishing process has been used for external surface finishing of Inconel 718 flat surfaces (5 mm thick plate). A number of experiments have been designed and conducted, to study the effect of three selected process parameters, i.e., processing time, pole rotation speed and weight percentage of abrasives, on percentage improvement in surface finish. An ANOVA has been used to analyze the experimental results. Experimental results indicate that, all the above mentioned parameters have considerable effect on surface finishing, of Inconel 718. Effect of different process parameters on surface microstructure has also been observed, using scanning electron microscopy.

KEYWORDS: Inconel 718, Magnetic Tool, Ferromagnetic Abrasive Particles, Magnetic Abrasive Finishing \& Percentage Improvement in Surface Finishing
\end{abstract}

Received: May 27, 2017; Accepted: Jun 19, 2017; Published: Jul 21, 2017; Paper Id.: IJMPERDAUG201723

\section{INTRODUCTION}

Inconel 718 is a nickel based alloy mainly used in the aircraft industry, steam turbine, Rocket motors due to its high thermal resistance and high tensile strength properties. Machining of Inconel 718 is very difficult due to its materialistic properties. While machining Inconel 718, cutting tool life was reduced and surface of the work piece, was also damaged [1,2]. Generally two types of tools are used for the cutting of Inconel 718: cemented carbide tools and ceramic tool [3]. Work surface, machined with these type of cutting tools damage the surface resulting in high surface roughness. So, different finishing processes such as lapping, honing, grinding are used to improve the quality of surface finish [4]. But these processes are not capable to produce the required surface finish on Inconel 718 surfaces.

A recently developed process, called Magnetic Abrasive Finishing (MAF) can also be used to finish very hard surface of Inconel 718. In MAF, the work piece is placed between north and South Pole of the magnet. Gap between both the poles of the magnet and the work piece is filled with magnetic, abrasive particles (MAP) that act as a finishing tool. These magnetic, abrasive particles aligned itself in the direction of magnetic force forming flexible magnetic abrasive brush. This magnetic, abrasive brush plays an important role for surface finishing [4]. Several researchers have utilized magnetic, abrasive finishing process for finishing of different material surface. Mori et al. (2003) performs the experiment over a stainless steel work piece to investigate the type of forces acting in MAF process. Observations indicate that three types of energies that are repulsive forces, tangential forces and normal forces are necessary for magnetization of abrasives [5]. Lin et al (2007) utilized MAF to perform free-form 
surface abrasion of stainless SUS304 stainless steel [6]. Kwak (2009), proposed a practical method to improve the magnetic flux density of non-ferrous materials (magnesium), as work piece in MAF process. It uses a permanent magnet on the opposite side of the work piece, to evaluate the results [7]. Kala \& Pandey (2014), perform the double disk MAF process for the flat surface of (copper alloy), work piece to check the effect of various parameters on finishing force and torque [8]. Sihag et al (2015), introduces a new MAF process, which uses a combination of both MAF and chemicaloxidation, for faster processing of surface finishing of work piece (tungsten). Experiments have been conducted to check the effect of various parameters such as work gap, concentration of oxidizing agent and weight of abrasives on tungsten work piece [9]. Wu.et al.(2015), studied different parameters like rotational speed of magnetic pole, grinding fluid and current frequency for a stainless steel plate, using a new method known as ultra-precision magnetic abrasive finishing [10].

In the present work, an attempt has been made to finish Inconel 718 flat surfaces with the magnetic abrasive finishing process, by utilizing a magnetic tool and another supporting magnet.

\section{EXPERIMENTAL SETUP}

An experimental setup has been designed for flat surface finishing of Inconel 718 surfaces (200x200x5 mm) with the help of rotating magnetic tool fixed in the vertical milling machine spindle. Magnetic tool is fabricated with aluminum $\operatorname{rod}(\varnothing 25 \times 125 \mathrm{~mm})$ and Nd-Fe-B Permanent Magnet of diameter $(\varnothing 20 \times 22 \mathrm{~mm})$.

Work piece (200x200x5 mm thick plate) is held on vertical milling machine bed chucks for finishing process. Movement of bed is controlled with the help of two hand wheels provided on both left and right side of the bed. A vertical head is positioned above the bed provided with a spindle to keep the magnetic tool in a vertical position above the work piece (Figure 1). Vertical milling machine is capable of rotating the spindle from 50 to $528 \mathrm{rpm}$. Two up and down lead screws are available to move the bed in upward and downward direction for maintaining the required gap between work piece and magnetic tool.

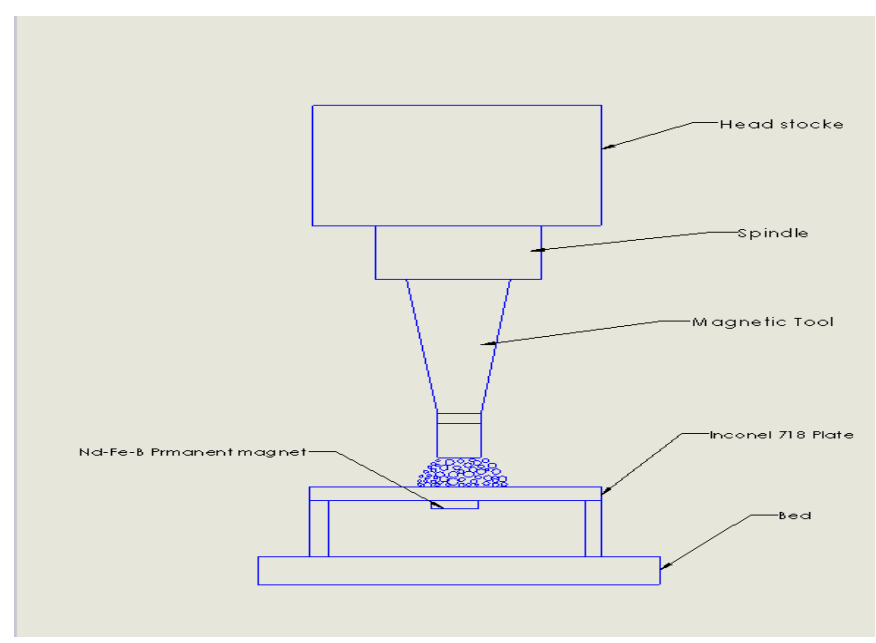

Figure 1: Schematic Diagram of Experimental Setup

Two wooden blocks have been used to keep the work piece slightly above the machine bed. With the help of suitable fixtures, work piece was held firmly on vertical milling machine bed. Another permanent magnet $(\varnothing 20 \times 5 \mathrm{~mm})$ was placed under the work surface to enhance magnetic flux density. Figure 2 shows the photographic view of experimental setup. 


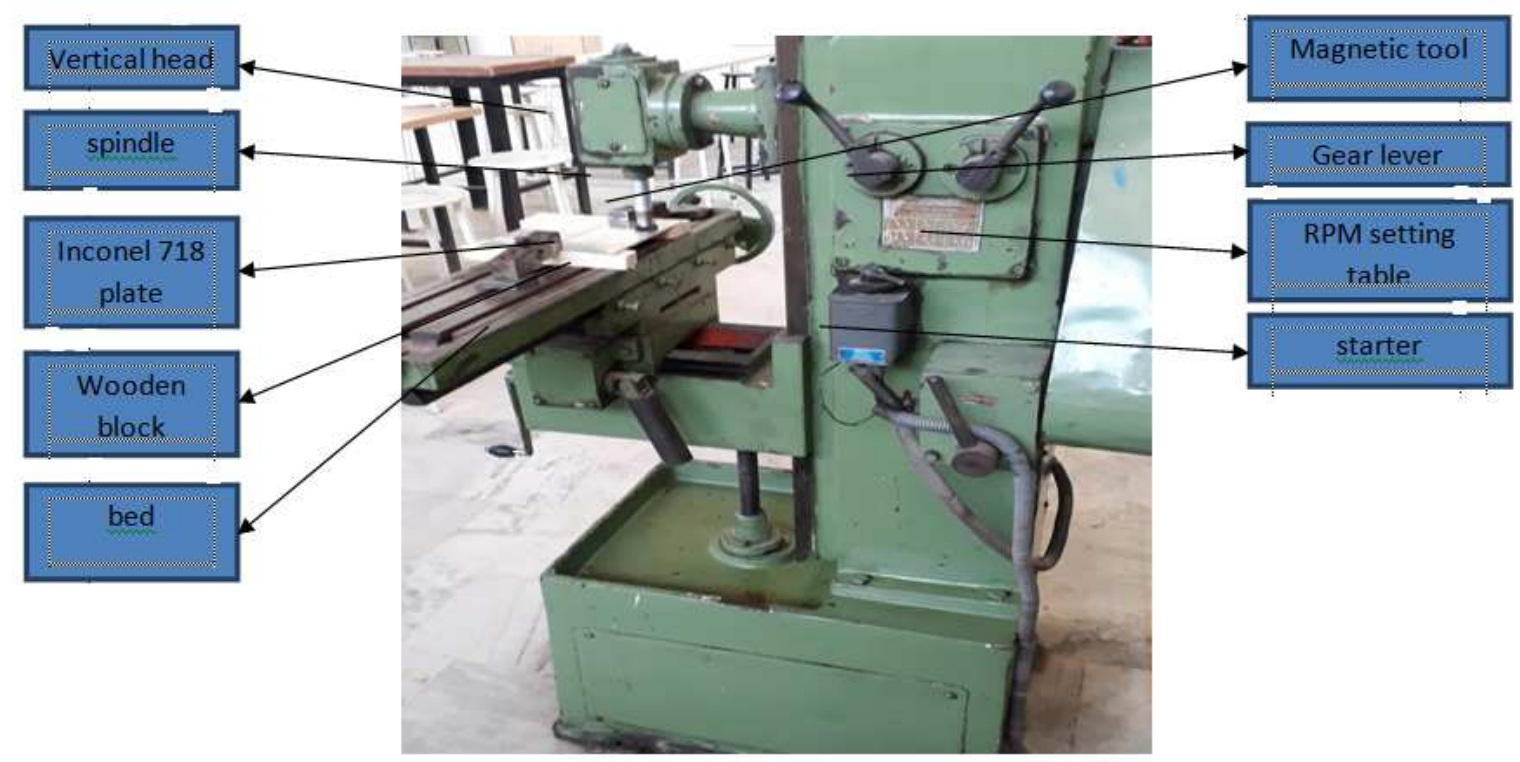

Figure 2: Photographic View of Experimental Set Up

\section{EXPERIMENTATION}

For finishing of Inconel 718 surface, work piece was held on vertical milling machine bed as shown in Figure 2. A magnetic tool is positioned in milling machine spindle and another permanent magnet is placed below the work piece. A strong magnetic field is induced between two magnets. Due to machining operation on Inconel 718 work piece surface, it has very rough surface with number of tool marks and asperities. Figure 3 shows SEM photograph of machine Inconel 718 surface which indicates lot of cutting tool marks and burrs. These are produced due to high cutting forces during machining of Inconel 718 surface.

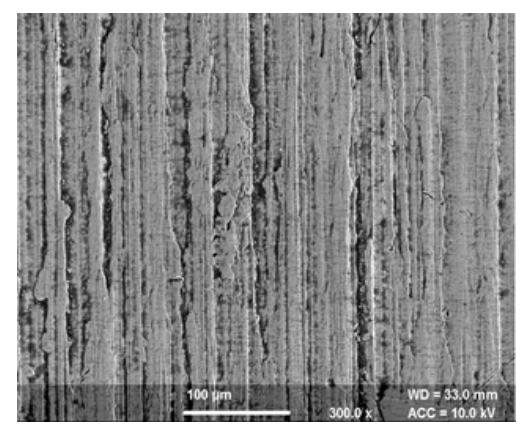

Figure 3: SEM photograph of Initial Surface

There are different process parameters which can influence the surface finishing process such as processing time, pole-work gap, pole rotational speed, quantity of abrasive particles, composition of ferromagnetic abrasive particles etc. On the basis of detailed literature survey and experimentation setup capabilities, three process parameters: Processing time, pole rotational speed, weight percentage of abrasive particles and their range have been selected for final surface finishing of Inconel 718 surface. Design-expert software has been used to plan the experiments. As per experimentation plan, seventeen experiments were conducted. 
A mixture of abrasive particles made up of tungsten carbide ( $660 \mu \mathrm{m}$ average particle size) and electrolyte iron particles $(\varnothing 300 \mu \mathrm{m}$ average particle size) is used as ferromagnetic abrasive particles for surface finishing of work surface. Soluble type Barrel finishing compound $(2 \mathrm{ml})$ has been used for each experiment. A gap of $3 \mathrm{~mm}$ is kept between magnetic tool and Inconel 718 plate and the gap is filled with ferromagnetic abrasive particles (3 gm). Due to high magnetic flux density between two magnets, a magnetic abrasive brush is formed over work surface of Inconel 718 plate that acts as a finishing tool while rotating over the surface and removing surface irregularities.

Table 1: Experimental Conditions

\begin{tabular}{|c|c|}
\hline Work piece & Inconel $718(200 \times 200 \times 5 \mathrm{~mm})$ \\
\hline Magnetic tool & Nd-Fe-B Permanent Magnet $(\varnothing 20 \times 22 \mathrm{~mm})$ \\
\hline Supporting Magnet & Nd-Fe-B Permanent Magnet $(\varnothing 20 \times 5 \mathrm{~mm})$ \\
\hline Range of Process parameters & $\begin{array}{l}\text { Processing Time }: 25-75 \mathrm{~min} \\
\text { Pole rotation speed }: 80-200 \mathrm{rpm} \\
\text { weight } \% \text { age of abrasive }: 25-45 \%\end{array}$ \\
\hline Abrasive Particles & Tungsten Carbide $(\varnothing 60 \mu \mathrm{m})$ \\
\hline Ferrous particles & Electrolytic Iron Particles $(\varnothing 300 \mu \mathrm{m})$ \\
\hline
\end{tabular}

Table 2: Coded and Real Level of Independent Variables

\begin{tabular}{|c|c|c|c|c|}
\hline \multirow{2}{*}{ Parameters } & \multirow{2}{*}{ Symbol } & \multicolumn{3}{|c|}{ Level } \\
\hline & & -1 & $\mathbf{0}$ & $\mathbf{1}$ \\
\hline Processing Time (Minutes) & A & 25 & 50 & 75 \\
\hline Pole Rotation speed( RPM) & $\mathrm{B}$ & 80 & 140 & 200 \\
\hline Weight \%age of Abrasives (\%age) & $\mathrm{C}$ & 25 & 35 & 45 \\
\hline \multicolumn{5}{|l|}{ Constant Parameters } \\
\hline Work piece Material & \multicolumn{4}{|c|}{ Inconel 718} \\
\hline Geometry of magnetic tool & \multicolumn{4}{|c|}{$\varnothing 20 \times 22 \mathrm{~mm}$} \\
\hline Dimensions of work piece & \multicolumn{4}{|c|}{$(200 \times 200 \times 5 \mathrm{~mm})$} \\
\hline
\end{tabular}

For final experimentation, vertical milling machine is used for finishing the flat surface of Inconel 718 $(200 \times 200 \times 5 \mathrm{~mm})$. Magnetic tool is held in vertical milling machine spindle and Inconel 718 plate is placed on the bed of milling machine. Gap between the magnetic tool and work surface is filled with ferromagnetic abrasive particles. Magnetic tool can be rotated according to experimentation plan given in Table 3.

In present work, Surface Response Methodology is used to design the experiments. Three parameters processing time, weight $\%$ of abrasive particles, pole rotation speed were selected and can be varied upto three levels (Table 2). After deciding parameters and their levels final experimentation plan was designed using ANOVA as shown in Table 3.

Table 3: Experimentation Plan

\begin{tabular}{|c|c|c|c|c|}
\hline Exp. No. & $\begin{array}{c}\text { Order of } \\
\text { Exp. }\end{array}$ & $\begin{array}{c}\text { Processing } \\
\text { Time }\end{array}$ & $\begin{array}{c}\text { Pole Rotation } \\
\text { Speed }\end{array}$ & $\begin{array}{c}\text { Weight \% of } \\
\text { Abrasive Particles }\end{array}$ \\
\hline 1 & 9 & 0 & -1 & -1 \\
\hline 2 & 11 & 0 & -1 & 1 \\
\hline 3 & 10 & 0 & 1 & -1 \\
\hline 4 & 17 & 0 & 0 & 0 \\
\hline 5 & 7 & -1 & 0 & 0 \\
\hline 6 & 16 & 0 & 0 & 0 \\
\hline 7 & 1 & -1 & -1 & 0 \\
\hline 8 & 13 & 0 & 0 & 0 \\
\hline 9 & 2 & 1 & -1 & \\
\hline
\end{tabular}




\begin{tabular}{|c|c|c|c|c|}
\hline \multicolumn{7}{|c|}{ Table 3: Contd., } \\
\hline 10 & 15 & 0 & 0 & 0 \\
\hline 11 & 14 & 0 & 0 & 0 \\
\hline 12 & 3 & -1 & 0 & 0 \\
\hline 13 & 5 & -1 & 0 & -1 \\
\hline 14 & 12 & 0 & 1 & -1 \\
\hline 15 & 6 & 1 & 0 & 0 \\
\hline 16 & 4 & 1 & 1 & 1 \\
\hline 17 & 8 & 1 & 0 & 1 \\
\hline
\end{tabular}

Seventeen experiments were conducted according to the experimental plan and work piece was taken out from the milling machine after each experiment. Next step was to measure the surface finish using surface roughness tester. Surface finish was measured at least three points to obtain final results for the surface finish. Final value of the surface finish was based on the average value of above three points and percentage of improvement in surface finishing (PISF) for all the seventeen experiments were calculated by comparing their average surface roughness values of unprocessed surface with processed surface. Initial value for surface roughness varies from $1.2 \mu \mathrm{m}$ to $3.14 \mu \mathrm{m}$ and final value for surface roughness varies from $0.05 \mu \mathrm{m}$ to $2.054 \mu \mathrm{m}$. Percentage improvement in surface finishing (PISF) for all the experiments was shown in Table 4.

Table 4: Experimental Responses

\begin{tabular}{|c|c|c|c|c|c|}
\hline \multirow{2}{*}{ Exp. No. } & \multirow{2}{*}{ Order of Exp. } & \multicolumn{5}{|c|}{ Independent Parameters (Coded) } & Responses \\
\cline { 3 - 6 } & & A & B & C & PISF \\
\hline 1 & 9 & 0 & -1 & -1 & 22 \\
\hline 2 & 11 & 0 & -1 & 1 & 29 \\
\hline 3 & 10 & 0 & 1 & -1 & 54 \\
\hline 4 & 17 & 0 & 0 & 0 & 50 \\
\hline 5 & 7 & -1 & 0 & 1 & 53 \\
\hline 6 & 16 & 0 & 0 & 0 & 43 \\
\hline 7 & 1 & -1 & -1 & 0 & 31 \\
\hline 8 & 13 & 0 & 0 & 0 & 44 \\
\hline 9 & 2 & 1 & -1 & 0 & 60 \\
\hline 10 & 15 & 0 & 0 & 0 & 40 \\
\hline 11 & 14 & 0 & 0 & 0 & 50 \\
\hline 12 & 3 & -1 & 1 & 0 & 45 \\
\hline 13 & 5 & -1 & 0 & -1 & 60 \\
\hline 14 & 12 & 0 & 1 & 1 & 34 \\
\hline 15 & 6 & 1 & 0 & -1 & 51 \\
\hline 16 & 4 & 1 & 1 & 0 & 47 \\
\hline 17 & 8 & 1 & 0 & 1 & 66 \\
\hline A- & Processing time B- pole rotation speed & & \\
\hline
\end{tabular}

\section{RESULTS AND DISCUSSIONS}

After performing seventeen experiments on the Inconel 718 plate according to experimental plan, each work surface was tested to check the percentage improvement of surface finishing. Design expert software is used to analysis the simultaneous effect of three different process parameters on percentage improvement of surface finish (PISF).

\section{Parametric Effect of Process Parameters on Percentage Improvement on Surface Finish}

Change in percentage improvement in surface finish by considering combination of any two process parameters is explained below: 


\section{Combined Effect of Processing Time and Pole Rotational Speed on PISF}

Figure 4shows the effect of simultaneous variation of processing time and pole rotational speed on the percentage improvement in surface finishing (PISF). At minimum processing time, -1 (25 minutes) and minimum pole-rotational speed -1(80 rpm), there is small increase in PISF. If only pole rotational speed is increased for minimum processing time, 1 (25 minutes), there is considerable increase in PISF. On the other hand, with the increase in processing time, initially there is small decrease in PISF and then further incremental trend in PISF is observed. It is observed that PISF is at the highest processing time, 1(75minutes) and medium pole rotational speed, $0(140 \mathrm{rpm})$. PISF increases with increase in processing time but start decreasing with increase in pole rotational speed. It may be due to the reason that with increase in processing time, magnetic tool with abrasive particles got some extra time for finishing resulting good surface finishing of Inconel 718 plate. But at high pole rotational speed, magnetic tool start losing its contact with Inconel 718 plate (work piece) resulting improper surface finishing.

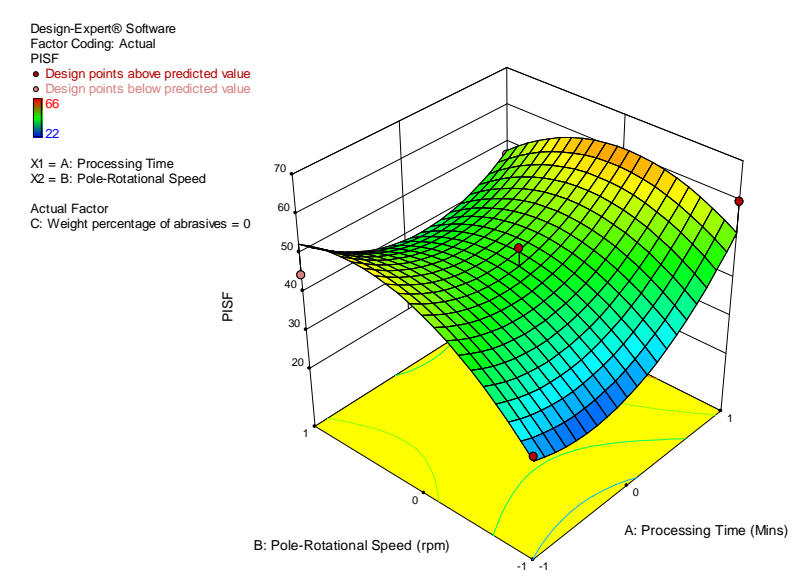

Figure 4: Combine Effect of Processing Time and Pole Rotational Speed on PISF

Figure $5 \mathrm{a}$ and $5 \mathrm{~b}$ indicates the variation of microstructure due to varition in processing time and pole rotaional speed. Figure 5a shows that with 75 min processing time and minimum pole rotational speed ( 80 rpm), work surface is free from tool marks with negligible burr. Figure $5 \mathrm{~b}$ shows at minimum processing time of 25 min and maxmium pole rotational speed(200), tool marks are observed on work suface. Peaks and valleys are still present on surface. It proves that for better surface finish, high processing time and low to medium pole rotational speed are preferable conditions.

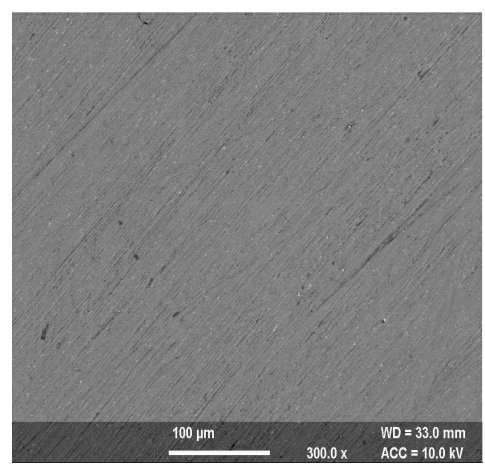

Figure 5 (a): SEM Photograph at Processing Condition: Processing Time:75min, Pole Rotation Speed: 80rpm, Weight \% of Abrasive Particles: 35 


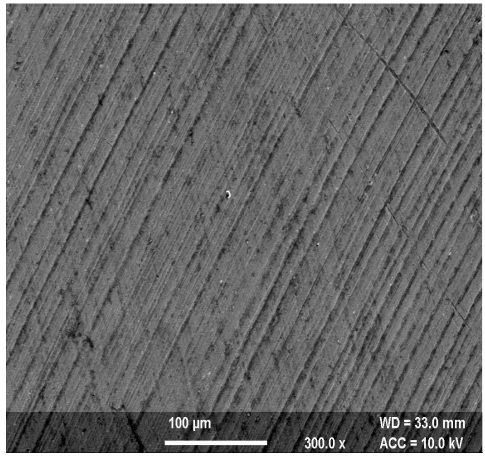

Figure 5(b): SEM Photograph at Processing conditionprocessing Time:25min, Pole Rotation Speed: 200rpm, Weight \% of Abrasive Particles: 35

\section{Combined Effect of Pole Rotational Speed and Weight \% Age of Abrasives}

Figure 6 shows the effect of simultaneous variation in pole rotational speed and weight \%age of abrasives on the percentage improvement in surface finishing (PISF). It is clear that PISF is minimum at smallest value of pole rotational speed,-1(80 rpm) and weight \%age of abrasives,-1(25\%). PISF increases with increase in pole rotational speed reaches the maximum value at maximum pole-rotational speed, 1 (200 rpm) and with minimum weight percentage of abrasives,-1(25 $\%)$. With simultaneous increase in pole rotational speed and weight percentage of abrasives, initially there is considerable increase in PISF and then PISF start decreasing with increase in weight percentage of abrasives. It may be due to the fact that with increase in weight percentage of abrasives, number of cutting edges which removes surface irregularities is increased. But as pole rotational speed is increased, it produces scratches on surface rather than finishing.

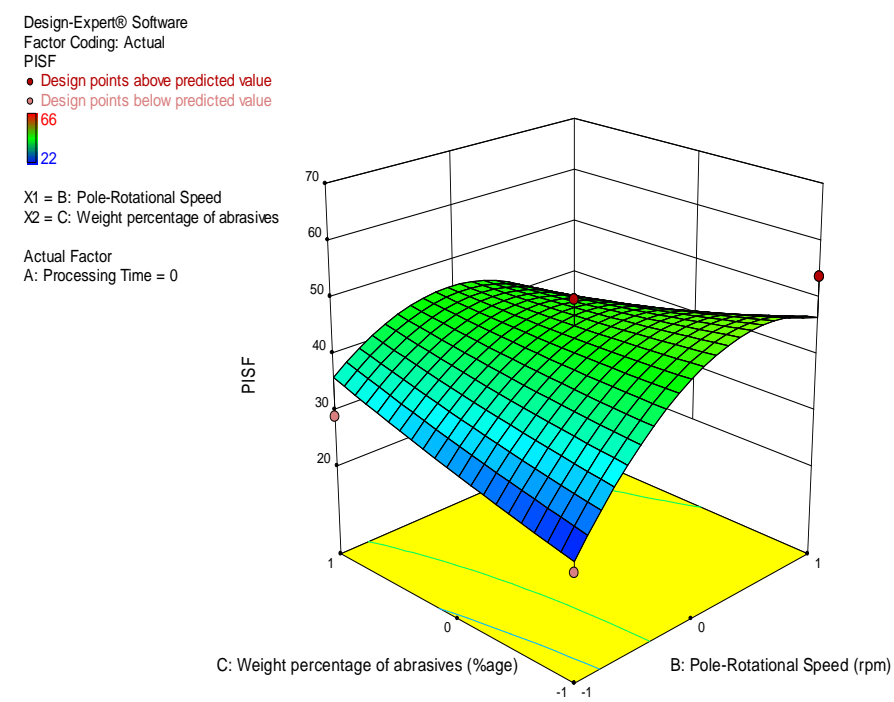

Figure 6: Combine Effect of Pole Rotational Speed and Weight \% Age of Abrasives 


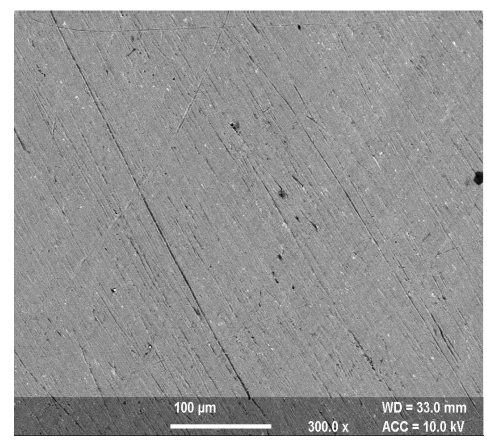

Figure 7(a): SEM Photograph at Processing Condition: Processing Time:50min, Pole Rotation Speed: 80rpm, Weight \% of Abrasive Particles: 45

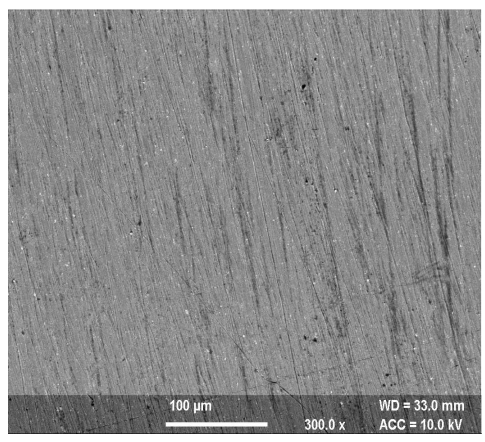

Figure 7(b): SEM Photograph at Processing Condition: Processing Time: 50min, Pole Rotation Speed: 200rpm, Weight \% of Abrasive Particles: 25

Figure 7(a) and 7(b) shows the SEM photographs of work surface with change in pole rotational speed and weight \%age of abrasive particles. Tool marks can be easily observed on work surface in both conditions. But comparatively lower pole rotational speed and higher weight percentage of abrasives gives better surface.

\section{Combined Effect of Weight \% Age of Abrasives and Processing Time}

Figure 8 shows the effect of simultaneous variation in processing time and weight \%age of abrasives on the percentage improvement in surface finishing (PISF). It is clear from Figure 8 that initially PISF decreases with increase in both processing time and weight \%age of abrasives and then increases with increase in processing time and weight \%age of abrasives. PISF is maximum at when both processing times and weight \%age of abrasives are at highest value (1). Reason behind such type of increase is that with high processing time and high weight \%age of abrasives, magnetic abrasive brush with high number of cutting edges initially removes the more material rather than finishing. And then further improves the surface finish as it keep on rotating of work surface for longer time by removing maximum of surface irregularities. 


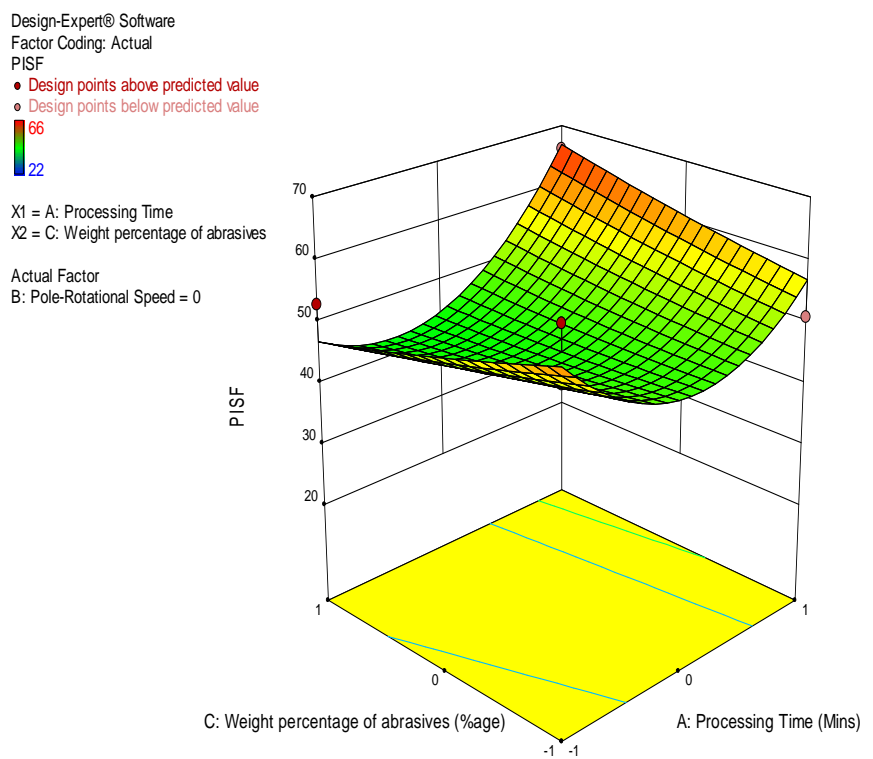

Figure 8: Combined Effect of Processing Time and Weight \% Age of Abrasives

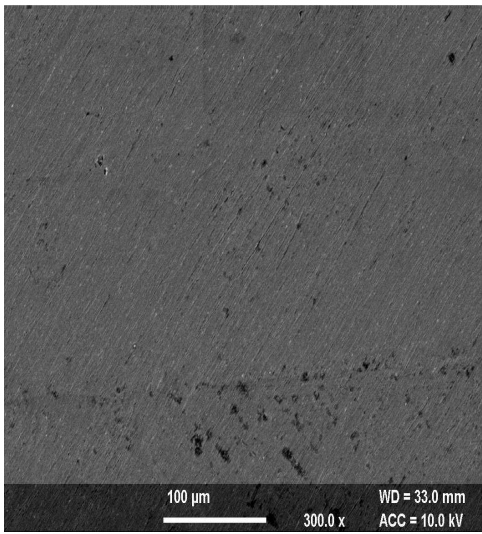

Figure 9(a): SEM Photograph at Processing Condition: Processing Time:75min, Pole Rotation Speed: 140rpm, Weight \% of Abrasive Particles: 45

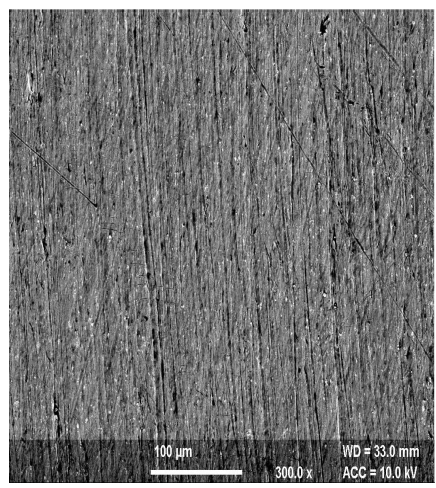

Figure 9 (b): SEM Photograph at Processing Condition: Processing Time:25min, Pole Rotation Speed: 140rpm, Weight \% of Abrasive Particles: 25 
Figure 9 (a) and 9 (b) shows the SEM photographs of work surface with change in processing time and weight \%age of abrasive particles. It can be observed that at higher processing time and higher weight \%age of abrasives gives better finish. But at lower processing time and lower weight \%age of abrasives, tool marks can be easily observed on work surface.

\section{CONCLUSIONS}

On the basis of experimental results obtained after magnetic abrasive finishing of Inconel 718 surface, following conclusions have been drawn:

- Inconel 718 surface can be successfully finished with magnetic abrasive finishing process by using tungsten carbide abrasives.

- All the three selected process parameters i.e. processing time, pole rotational speed and weight percentage of abrasive particles considerably influence the finishing process.

- Processing time is a major process parameter which influences PISF. As compared to small processing time, -1 (25mins), better surface finish can be achieved if the surface is processed for medium, 0 (50 minutes) to longer durations 1(75 minutes).

- Effect of surface rotational speed is related with other process parameters. But PISF is better at medium to higher surface rotational speeds.

- PISF is considerably influenced weight \%age of abrasive particles but its effect is related with other two parameters. Still it can be concluded that medium to larger weight percentage of abrasive particle gives better surface finish.

\section{REFERENCES}

1. Ezugwu, E.O., Wang, Z. M., \& Machado, A. R. (1999). The machinability of nickel-based alloys: a review. Journal of Materials Processing Technology, 86, 1-16.

2. Arunachalam, R., \& Mannan, M.A. (2000). Machinability of nickel-based high temperature alloys. Machining Science and Technology, 4 (1), 127-168.

3. Dudzinski, D., Devillez, V., Moufkia, V., Larrouque re, D., Zerrouki, V., \&Vigneau, J. (2004). A review of developments towards dry and high speed machining of Inconel 718 alloy. International Journal of Machine Tools \& Manufacture, 44, 439456.

4. Jayswal, S. C., Jain, V. K., \&Dixit, P. M. (2005). Magnetic Abrasive Finishing Process - a parametric analysis. journal of advanced manufacturing systems, 4(2), 131-150.

5. Mori, T., Hirota, K., \& Kawashima, Y. (2003). Clarification of Magnetic Abrasive Finishing Mechanism. Journal of Materials Processing Technology, 143-144, 682-686.

6. Lin, C.T., Yang, L.D., \& Chow, H.M. (2007). Study of Magnetic Abrasive Finishing in Free-Form Surface Operations using The Taguchi Method. International Journal of Advance Manufacturing Technology, 34, 122-130.

7. Kwak, J. S. (2009). Enhanced magnetic abrasive polishing of non-ferrous metals utilizing a permanent magnet. International Journal of Machine Tools \& Manufacture, 49, 613-618. 
8. Kala, P., \&Pandey, P. M. (2014). Experimental Study on Finishing Forces In Double Disk Magnetic Abrasive Finishing Process While Finishing Paramagnetic Work piece. Procedia Materials Science, 5, 1677 - 1684.

9. Sihag, N., Kala, P., \& Pandey, P. M. (2015). Chemo Assisted Magnetic Abrasive Finishing. Experimental Investigation Procedia CIRP, 26, $539-543$.

10. Wu, J., Zou, Y., \& Sugiyama, H. (2015). Study on ultra -precision magnetic abrasive finishing process using low frequency alternating magnetic field. Journal of Magnetism and Magnetic Materials, 386, 50-59. 
